\title{
Intraarterial Infusion of Lipiodol CDDP Suspension into the Internal Iliac Artery — Pre Clinical Dose Escalation Study Using Canine Model
}

\author{
Hiroshi Okazaki, ${ }^{1}$ Kohei Kurokawa, ${ }^{2}$ Kazuhiro Suzuki, ${ }^{2}$ \\ Yuka Matsumura, ${ }^{3}$ Shigeru Honda, ${ }^{3}$ Ryuya Horiuchi, ${ }^{3}$ \\ and Hidetoshi Yamanaka ${ }^{2}$
}

\begin{abstract}
Background and Aims : To investigate the usefulness of intraarterial chemotherapy (IAC) with CDDP powder and lipiodol suspension (LCS) for invasive bladder cancer as a dose escalation modality of conventional IAC, we administered LCS into the internal iliac artery of dogs and evaluated pharmacokinetics of CDDP. Methods : An angiographic catheter was inserted to the internal iliac artery of an anesthetized dog under fluoroscopic guidance. CDDP solution, LCS, or a combination of CDDP solution and LCS were intra-arterially infused. For comparison, CDDP solution was also intravenously infused. The dose of CDDP ranged from 1 to $3 \mathrm{mg} / \mathrm{kg}$. For combination study, the dose of CDDP ranged from 3 to $5 \mathrm{mg} / \mathrm{kg}$. In all groups, blood samples were collected 5, 15, 30, 60, 120, and 180 minutes after administration, and serum Pt levels were measured. Results : In the LCS group, the serum Pt level was lower than those after intravenous or intraarterial infusion of CDDP solution. In the combination therapy group, the serum Pt level increased with the total dose of CDDP. Conclusions : Intraarterial infusion of LCS showed a lower systemic CDDP level. This method may reduce the incidence of side effects, suggesting the usefulness of intraarterial infusion therapy with LCS for invasive bladder cancer.

(Kitakanto Med J $2003 ; 53: 275 \sim 279$ )
\end{abstract}

Key words : invasive bladder cancer, CDDP, Lipiodol, intraarterial, chemotherapy

\section{Introduction}

Previous studies have reported the usefulness of intraarterial infusion chemotherapy (IAC) as a combination therapy for invasive bladder cancer. ${ }^{1}$ This therapy may increase administered concentrations of anticancer agents that act on tumor tissues. Cisplatin (cis-diamminodichloroplatinum, CDDP) has been used most widely as a single agent or in combination regimens. ${ }^{2,3}$

To treat invasive bladder cancer, we performed super-selective intraarterial infusion chemotherapy (SSIAC) in the Department of Urology, Gunmla University School of Medicine. SSIAC targets the tumor site alone, and increase the tissue concentrations of anticancer agents compared to standard IAC by infusing anticancer agents into tumor-nourishing blood vessels alone, exhibiting enhanced antitumor effects (Fig. 1 ). As an anticancer agent, the CDDP solution was used.

To improve the actions of anticancer agents, they should be present in tumor tissues at a higher concentration for a long duration. ${ }^{4,5}$ We investigated intraarterial infusion of CDDP using lipiodol as a carrier. IAC using lipiodol, which is an oil contrast medium, as a carrier has commonly been administered to patients with liver cancer, utilizing selective tumor accumulation. ${ }^{6,7}$ Good results have been reported. ${ }^{8}$

In this study, we prepared lipiodol cisplatin suspension (LCS) containing CDDP to maintain CDDP in

1 Department of Urology, Tatebayashi Kosei Hospital, 262-1, Narushima-machi, Tatebayashi, Gunma, 374-8533, Japan

2 Department of Urology, Gunma University School of Medicine

3 Department of Pharmacy, Gunma University School of Medicine

Received : June 4, 2003

Address : HIROSHI OKAZAKI Department of Urology, Tatebayashi Kosei Hospital, 262-1, Narushima-machi, Tatebayashi, Gunma,

374-8533, Japan 


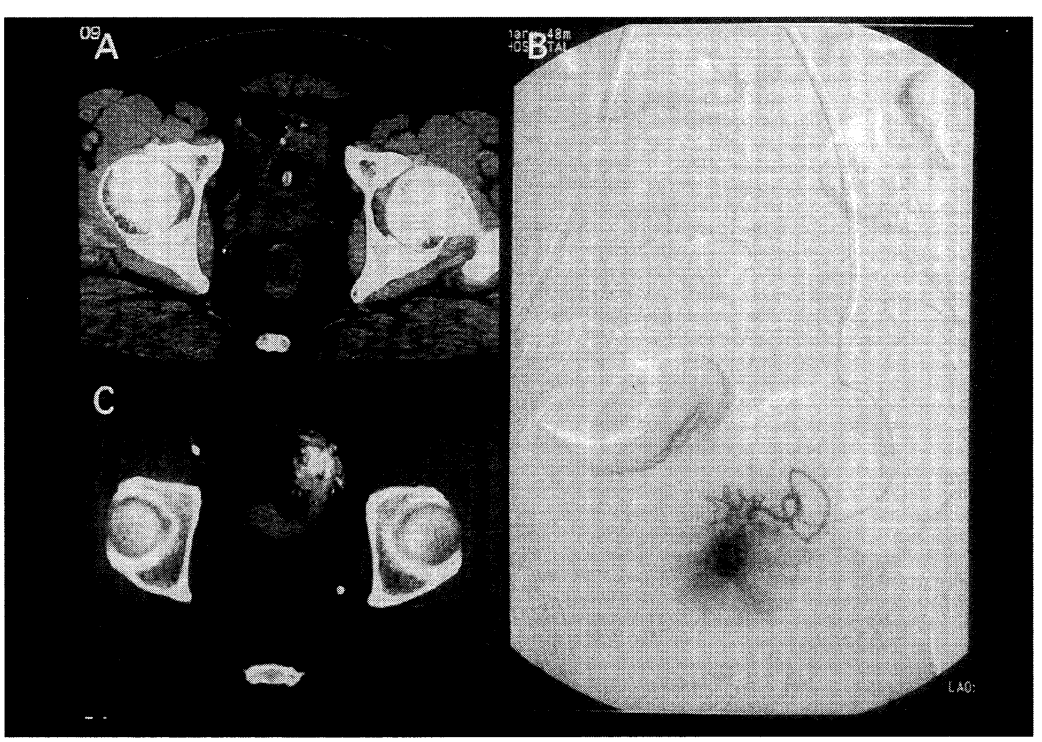

Fig. 1 48-year-old, male, TCC G2, T2

A : Non-enhanced CT of the bladder

B : Super selective angiography image shows an enhancing tumor and a 3-Fr Microferret catheter inserted into tumor-nourishing vessel from the superior vesical artery

$\mathrm{C}$ : Angio-CT image shows an enhancing tumor

tumor tissues for a long duration and obtained enhanced antitumor effects, utilizing selective intra-tumor retention of lipiodol.

A basic experiment was performed to investigate whether SSIAC facilitates selective infusion of LCS to the bladder tumor site. We infused LCS into the internal iliac artery of dogs, and compared the pharmacokinetics of CDDP to those after arterial or intravenous infusion of CDDP solution.

\section{Materials and Methods}

We used two types of CDDP, CDDP solution and LCS. In preparing LCS, CDDP in its original powder form (served by Nihon Kayaku Co., Ltd. Tokyo.) was initially crushed in an agate bowl for five minutes, and mixed with lipiodol, an oil contrast medium, using an ultrasound washer for five minutes. LipiodolCDDP-suspension at $50 \mathrm{mg} / \mathrm{ml}$ was used. The maximum diameter of CDDP crystals was $20 \mu \mathrm{m}$ under a light microscope (Fig. 2 ).

With respect to the administration method, we established the following four groups :

Group A : Intravenous infusion of CDDP solution (1, $2,3 \mathrm{mg} / \mathrm{kg}$ )

Group B : Intraarterial infusion of CDDP solution (1, $2,3 \mathrm{mg} / \mathrm{kg}$ )

Group C: Intraarterial infusion of $\operatorname{LCS}(1,2,3 \mathrm{mg} /$ $\mathrm{kg})$

Group D : Combination intraarterial infusion therapy with LCS and CDDP solution $(3,4,5 \mathrm{mg}$ / $\mathrm{kg})$.

Experimental procedure; Two adult dogs with ranging from 10 to $16 \mathrm{~kg}$ in weight were used for the same

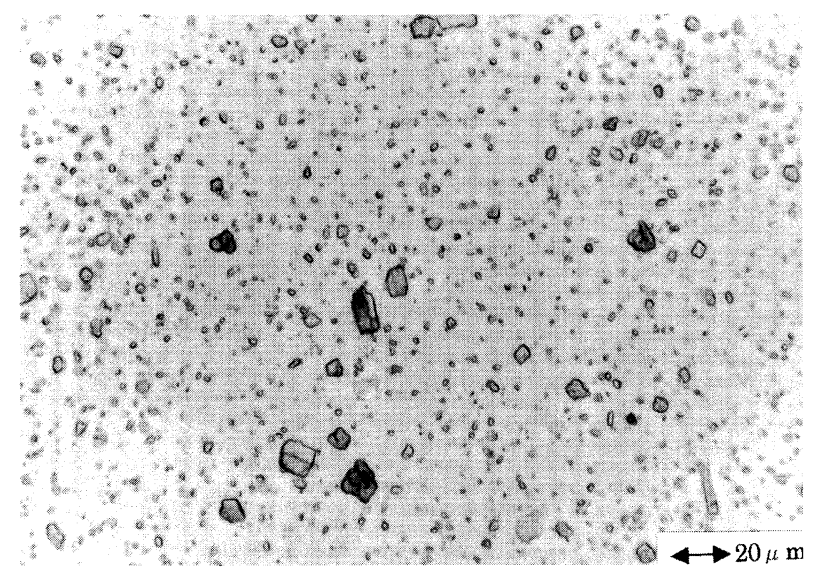

Fig. 2 Image of LCS under a light microscope $(\times 400)$

administration. All animals were cared for in accordance with the guideline set forth by the "Guide for the Care and Use of Laboratory Animals" published by the National Institutes of Health (NIH Publ. No. 86-23, revised 1985). This experimental study was performed with the approval of the Animal Care and Experimental Committee, Gunma University, Showa Campus. Under general anesthesia, a Teflon needle was inserted into the anterior limb vein for continuous intravenous drip of physiological saline. In group A, CDDP solution was infused for five minutes after infusion of physiological saline. In group B and C, the left internal carotid artery was exposed, and a $5 \mathrm{Fr}$. angiographic catheter was inserted into the internal iliac artery using a guide wire under fluoroscopy. After confirming the catheter end being present in the internal iliac artery by the enhanced bladder of infused contrast medium, CDDP solution and LCS were in- 
Table 1 Mean serum Pt levels

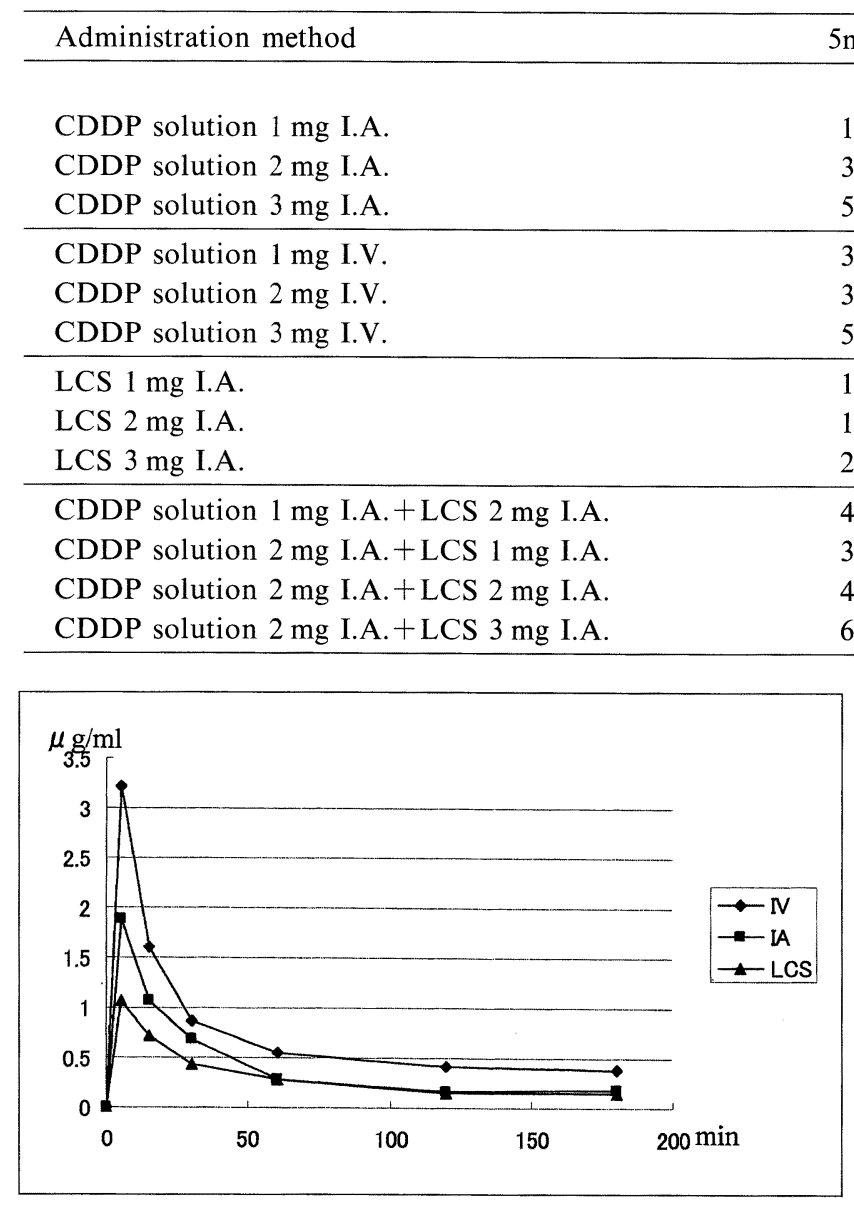

Fig. 3 Mean serum Pt levels doses of $1 \mathrm{mg} / \mathrm{kg}$

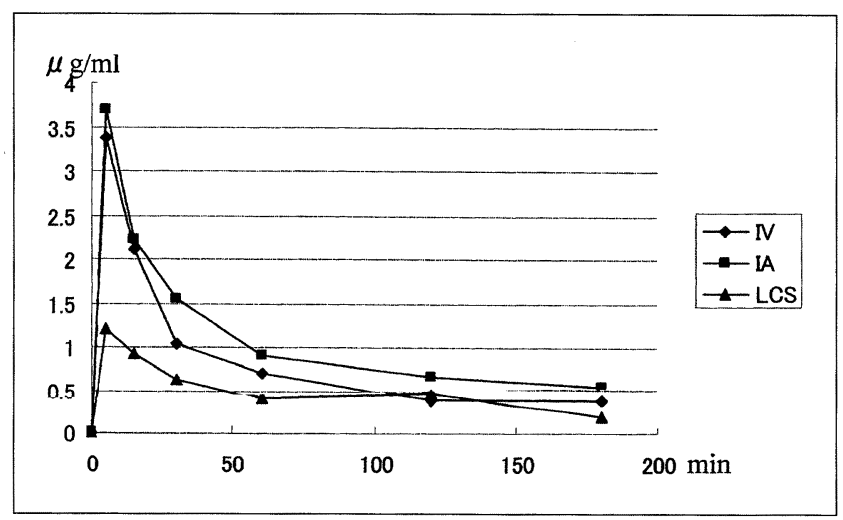

Fig. 4 Mean serum Pt levels doses of $2 \mathrm{mg} / \mathrm{kg}$

fused for five minutes. The catheter was thoroughly washed out in physiological saline so that there was no residue of the agents in the catheter. For group $\mathrm{D}$, the CDDP solution was initially infused, followed by LCS. Peripheral blood samples were collected 5, 15, $30,60,120$, and 180 minutes after infusion of the agents, and centrifuged. Serum samples were snapfrozen and stored. During the experiment, a rapid continuous intravenous drip of physiological saline was administered. The transfusion volume was $2 \mathrm{ml} /$ $\mathrm{kg} / \mathrm{min}$. CDDP was measured by atomic absorption

\begin{tabular}{lccccc}
5 min & $15 \mathrm{~min}$ & $30 \mathrm{~min}$ & $60 \mathrm{~min}$ & $120 \mathrm{~min}$ & $180 \mathrm{~min}$ \\
\hline \multicolumn{5}{c}{ Serum Pt levels $(\mu \mathrm{g} / \mathrm{ml})$} \\
3.89 & 1.06 & 0.69 & 0.28 & 0.18 & 0.18 \\
5.01 & 2.23 & 1.56 & 0.91 & 0.67 & 0.56 \\
3.22 & 3.17 & 2.04 & 1.16 & 0.79 & 0.76 \\
3.37 & 1.60 & 0.87 & 0.55 & 0.42 & 0.39 \\
5.48 & 2.12 & 1.03 & 0.70 & 0.38 & 0.38 \\
1.08 & 3.86 & 2.59 & 1.51 & 0.87 & 0.73 \\
1.20 & 0.72 & 0.43 & 0.28 & 0.16 & 0.16 \\
2.73 & 0.93 & 0.63 & 0.40 & 0.45 & 0.19 \\
4.00 & 2.23 & 1.54 & 0.96 & 0.50 & 0.37 \\
3.16 & 2.72 & 1.82 & 1.05 & 0.69 & 0.67 \\
4.18 & 2.25 & 1.75 & 1.25 & 0.95 & 0.94 \\
6.04 & 3.20 & 2.45 & 1.64 & 1.03 & 0.82 \\
& 5.01 & 3.64 & 2.70 & 2.13 & 2.10 \\
\hline
\end{tabular}

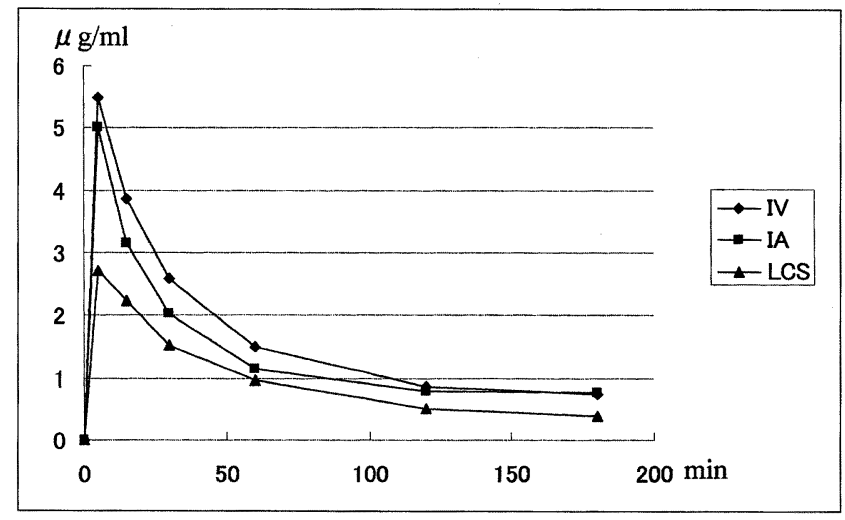

Fig. 5 Mean serum Pt levels doses of $3 \mathrm{mg} / \mathrm{kg}$

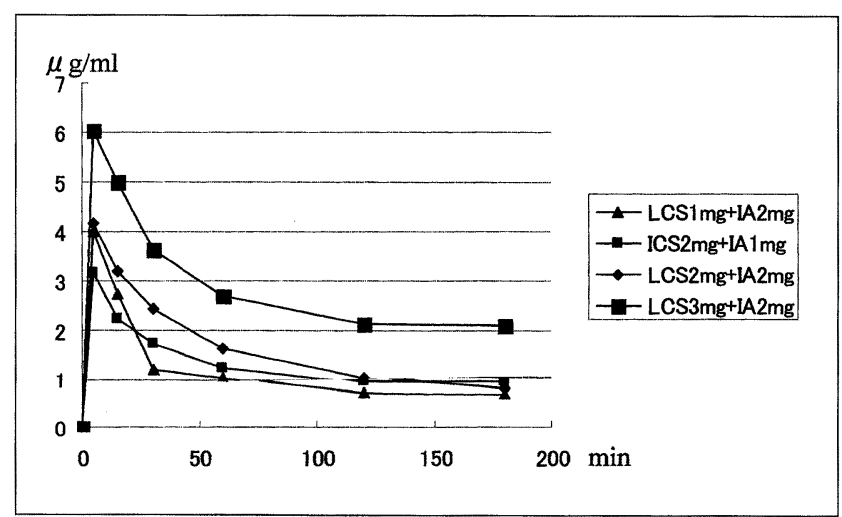

Fig. 6 Mean serum Pt levels in the IA + LCS group doses of 3-5 $\mathrm{mg} / \mathrm{kg}$

analysis (Z-8000 polarization Zeman atomic absorption spectrophotometer, Hitachi) using $\mathrm{Pt}$ as a marker. The mean serum Pt levels were compared among the 3 administration methods.

\section{Results}

Serial changes in the mean Pt level with respect to the doses of CDDP are shown in Table 1 and Figs. 3 to 6. With respect to the administration method, the serum Pt level in the LCS group at all doses was lower than those in the IV and IA groups. In the IA + LCS 
group, the serum Pt level depended on the total dose. In dogs studied with IA + LCS at a total dose of $3 \mathrm{mg} /$ $\mathrm{kg}$, the serum Pt level was slightly higher than that in dogs treated with $3 \mathrm{mg}$ of LCS.

\section{Discussion}

Intraarterial infusion therapy for invasive bladder cancer has been administered previously. ${ }^{9 \sim 12}$ This therapy produces an increase in the local tissue level of the drug delivered to the tumor site, higher local peak concentration and an increased area under the curve. To treat invasive bladder cancer, super-selective intraarterial infusion chemotherapy (SSIAC) that selectively infuses the anticancer agents is administered in the Department of Urology, Gunma University School of Medicine. In this therapy, anticancer agents are infused targeting the tumor site alone, rather than standard intraarterial infusion therapy. In this method, a 3 Fr.-microferret catheter is inserted via the lumen of a standard catheter, and CDDP is selectively infused into tumor-nourishing blood vessels. Therefore, this procedure may facilitate the maintenance of higher tissue concentrations of anticancer agents than routine intraarterial infusion therapy, showing more potent antitumor effects. Thirty-six patients with invasive bladder cancer (T2-4) were treated with an intraarterial infusion of CDDP under super selective angiography. The response rate $(\mathrm{CR}+\mathrm{PR})$ was $72 \%$ $(26 / 36)$.

To infuse a high concentration of CDDP for a dose escalation study, we used LCS that was prepared by mixing lipiodol, an oil contrast medium which persists in tissues for a long duration, with CDDP.

The efficacy of lipiodol in patients with hepatocellular carcinoma has been reported. ${ }^{13}$ Selective intratumor accumulation of lipiodol is associated with the presence of neogenetic tumor blood vessels. This finding has also been reported in patients with lung cancer, pancreatic cancer, and gallbladder cancer. ${ }^{14}$

Intraarterial infusion of anticancer agents using lipiodol as a carrier has been reported in many patients with hepatocellular carcinoma. ${ }^{15 \sim 18}$ When lipiodol is mixed with CDDP, lipiodol stays at the peripheral level, and CDDP also stays for long time. Sasaki et al. reported that intraarterial infusion of CDDP and LCS resulted in complete tumor necrosis in eight of ten patients with hepatocellular carcinoma. ${ }^{15}$ Yamamoto et al. also performed intraarterial infusion of LCS in patients with hepatocellular carcinoma, and reported that 1-year and 3-year survival rates were $89.84 \%$ and $68.51 \%$, respectively, achieving a good treatment response. ${ }^{16}$ With respect to tissue Pt levels, Shibata et al. measured liver Pt levels after intraarterial infusion of LCS in eight patients with hepatocellular car- cinoma, and reported that the Pt level in the cancer site was 21.8 -fold that in the non-cancer site, suggesting selective accumulation in the tumor site. ${ }^{17}$ LCS containing lipiodol as a carrier selectively delivers CDDP to the tumor site alone.

Only Yamashita et al. reported LCS for bladder cancer. ${ }^{19}$ They performed intraarterial infusion of LCS in six patients with invasive bladder cancer, and reported that $\mathrm{CR}, \mathrm{PR}$, and $\mathrm{MR}$ were achieved in two, three, and one patient, respectively. However, two patients developed dermal ulcer in the gluteal region. This was because a catheter could not be selectively inserted into the vesical artery, so the agent was infused through the internal iliac artery. SSIAC facilitates selective infusion of CDDP into tumor-nourishing blood vessels alone, and inflow to the inferior gluteal artery can be avoided. This procedure facilitates LCS delivery to the tumor site alone.

In this experiment using dogs, the serum Pt level after infusion of LCS was lower than those after intraarterial or intravenous infusion of CDDP solution. Furthermore, there was no marked difference between intraarterial infusion and intravenous infusion. Therefore, systemic side effects should be considered, as indicated for intravenous infusion. LCS shows affinity for the target organ via lipiodol, and is present in tumor tissues for a long duration, exhibiting potent antitumor effects. Furthermore, a decrease in the systemic circulation volume may relieve side effects. These results suggest the usefulness of adding LCS infusion to standard SSIAC.

\section{Acknowledgements}

We thank Nack Co., Takasaki for measurement of serum Pt concentration.

\section{References}

1. Galetti TP, Pontes JE, Montie J, et al. Neoadjuvant intra-arterial chemotherapy in the treatment of advanced transitional cell carcinoma of the bladder: Results and follow up. J Urol 1989 ; 142: 1211-1215.

2. Mokarim A, Uetani M, Sakamoto I, et al. Transarteterial infusion of cisplatin and doxorubicin in bladder cancer. Acta Oncol 1997; 36: 175181.

3. Stewart DJ, Eapen L, Hirte WE, et al. Intraarterial cisplatin for bladder cancer. J Urol $1987 ; 138: 302-305$.

4. Chen HS, Gross JF. Intra-arterial infusion of anticancer drugs: Theoretic aspects of drug delivery and review of response. Cancer Treat Rep $1980 ; 64: 31-40$.

5. Terashima Y. CDDP concentration of bladder 
cancer-Comparison between intraarterial and intravenous infusion- J Jpn Soc Cancer Ther $1988 ; 23: 859-866$.

6. Nakamura K, Tashiro S, Hiraoka $\mathrm{T}$, et al. Hepatocellular carcinoma and metastatic cancer detected by lodized Oil. Radiology 1985; 16 : 15-17.

7. Yumoto $\mathrm{Y}$, Jinno K, Tokuyama K, et al. Hepatocellular carcinoma detected by lodized oil. Radiology $1985 ; 16: 19-29$.

8. Kanematsu $\mathrm{T}$, Inokuchi $\mathrm{K}$, Sugimachi $\mathrm{K}$ et al. Selective effects of lipiodolezed antitumor agents. J Sur Oncology $1984 ; 25$ : 218-226.

9. Noguchi R, Miyanaga N, Koiso K, et al. Intraarterial chemotherapy for bladder cancer by insertion of catheter from inferior gluteal artery. Urol Int $1991 ; 47: 113-115$.

10. Schulman CC, Wespes E, Delcour C, et al. Intra-arterial chemotherapy of infiltrative bladder carcinoma. Eur Urol 1985; 11: 220-223.

11. Chechile G, Montie J, Pontes JE, et al. Neoadjuvant intra-arterial chemotherapy in locally advanced bladder cancer. Prog Clin Biol Res $1990 ; 353$ : 153-160.

12. Jacobs SC, Menashe DS, Mewissen MW, et al. Intraarterial cisplatin infusion in the management of the transitional carcinoma of the bladder. Cancer $1989 ; 64: 388-391$.

13. Bronowicki JP, Vetter D, Dumas F, et al. Transcatheter oily chemoembolization for hepatocellular carcinoma. A 4-year study of 127 French patients. Cancer 1994; $74: 16-24$.
14. Konno $\mathrm{T}$, Maeda $\mathrm{H}$, Iwai $\mathrm{K}$, et al. Selective targeting of anti-cancer drug and simultaneous image enhancement in solid tumors by arterially administered lipid contrast medium. Cancer $1984 ; 54$ : 2367-2374.

15. Sasaki $H$, Imaoka $S$, Iwanaga $T$, et al. New approach of chemoemblization for hepatocellular carcinoma -Lipiodol, Cisplatin Sandwitch therapy- J Jpn Soc Cancer Ther 1986; 21 : 65-72.

16. Yamamoto $\mathrm{K}$, Shimizu T, Narabayashi $\mathrm{I}$. Intraarterial infusion chemotherapy with Lipiodol-CDDP Suspension for hepatocellular carcinoma. Cardiovasc Intervent Radiol 2000 ; 23 : 26-39.

17. Shibata J, Kimura S, Chikazawa H, et al. Intrahepatic artery infusional chemotherapy with Cisplatin-Suspension in lipiodol (LPS) for hepatocellular carcinoma (II) -A clinical study-. J Jpn Soc Cancer Ther 1988; 23 : 2750-2759.

18. Kamada K, Nakanishi T, Kitamoto $M$, et al. Long-term prognosis of patients undergoing transcatheter arterial chemoembolization for unresectable hepatocellular carcinoma : comparison of cisplatin lipiodol suspension and doxorubicin hydrochloride emulsion. J Vasc Interv Radiol $2001 ; 12$ : 847-854.

19. Yamashita $\mathrm{S}$, Kanetake H, Saito $\mathrm{Y}$, et al. Intraarterial chemotherapy with CisplatinPhosphatidylcholine-Lipiodol Suspension (CPLS) for invasive bladder cancer. Jpn J Cancer Chemother 1992;19: 1087-1089. 